\title{
Illumination Correction from Psoriasis Image Data
}

\author{
Gabriela Maletti and Bjarne Ersbøll \\ Department of Informatics and Mathematical Modelling, \\ T echnical Unirersity of Denmark, \\ DK-2800 Kgs. Lyngby, Denmark \\ \{gmm, be\}@imm.du.dk \\ http://www.imm.dtu.dk/image
}

\begin{abstract}
An approach to automatically correct illumination problems in dermatological images is presented. The illumination function is estimated after combining the thematic map indicating skin -produced by an automated classification scheme- with the dermatological image data. The user is only required to specify which class has the most suitable thematic map to use in the illumination correction. Results are shown for real examples. It is also shown that the classification output improves after illumination correction.
\end{abstract}

\section{Introduction}

We use a set of $175 R G B$ psoriasis lesion images, of size 556 pixels by 748 pixels, tak en at the Gentofte Hospital, Denmark, during pilot sessions with three invited patients. For each patient, three lesions were follo w ed once a during at least three weeks. In each session, five images of each lesion were taken. Examples can be seen in Figure 2. The skin images are affected by shadows due to the non-planar shape of the objects. Thanks to the use of an integrating sphere [1] (see Figure 1) with optimal illumination conditions most of the skin images could be corrected. Normally, the sphere is used under the assumption that the captured objects are plane. Here, a quadratic model is assumed for the objects, because for instance an arm or a leg, as a first approximation, is more similar to a cylinder than to a plane.

On the other hand, in an ideal situation, for a given lesion, the classification output should contain a repeated pattern within and betw een sessions. It vould be preferable to have an algorithm which automatically selects the objects to be aligned from the thematic maps. How ever, as itwas reported in [5], for many examples without an y illumination correction, this is not a possibility. Thus, alignment and registration could turn to be a difficult -if not impossible- task. In order to be able to produce valid results regarding the actual data-set, an illumination correction scheme is proposed here. A reference for RGB and HSV values for normal skin can be found in [7]. In this work a method for analysis of color $\mathrm{c}$ hanges due to pathoplysiologic processes in the skin was reported. 

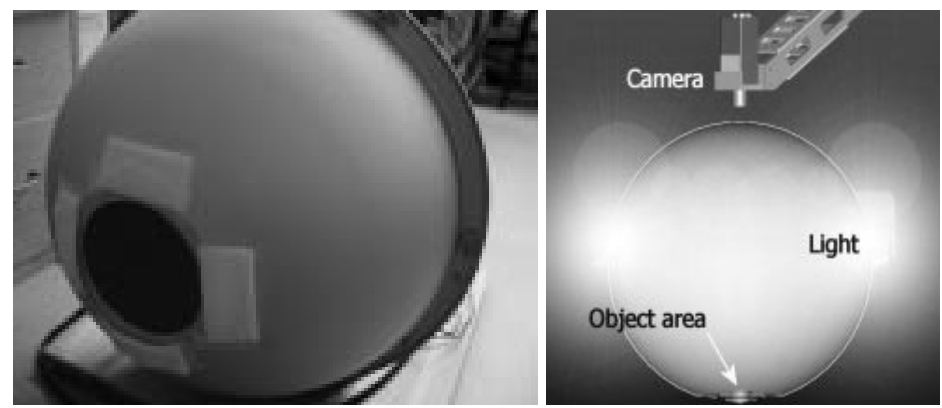

Fig. 1. Left: Integrating sphere with optimal illumination conditions used to capture the images. Right: Sketch of the sphere integrating lighting technology with camera technology. Figure courtesy of Videometer A/S [1].

\section{Illumination Correction Scheme}

A temporal set of images for which it can be assumed that the data in corresponding neighborhoods has the same distribution is required. The images of lesions with psoriasis can be assumed to contain three classes: background, normal skin and lesion. In order to segment the lesions, a two-stage hierarchical classification scheme has been proposed in [4]. The user is required to select the class which he considers most suitable to be used as input to the Illumination Function Estimation scheme. Neighborhoods of pixels belonging to that class are assumed to satisfy the same homogeneity criteria. Data are interpolated and the illumination function is estimated from these data. Details follow.

\subsection{Classification Scheme}

For completeness, general aspects of the scheme proposed in [5] are given here. Basically, in the first stage, the skin is separated from the background, and, in the second stage, the lesion is separated from the normal skin. In both stages, the classes are assumed to be Gaussian distributed. In each stage, means, standard deviations and prior probabilities of the classes were estimated with an Expectation-Maximization Algorithm [9]. These statistics were introduced in a discrimination function [2], which was applied to the output of the convolution of the image with a circular window of optimal size [6].

\subsection{Illumination Function Estimation}

Following [3], let $i(x, y)$ be the illumination function affecting the original scene $f(x, y)$ :

$$
g(x, y)=i(x, y) f(x, y)
$$


such that $g(x, y)$ is the observed illumination affected scene. This model assumes that the output is the product between the amount of arriving light and the original scene reflectivity. Basically, the region representing a used selected class $k$ is used to model the illumination function. Within the $\mathrm{k}$-th class we assume as a first approximation that the original image should be constant:

$$
i(x, y)=\frac{g_{k}(x, y)}{\beta_{k}}
$$

where $g_{k}$ is the observed illumination affected scene in the region representing the class $k$, and $\beta_{k}$ is a unknown constant.

Pixel values belonging to the region representing the $\mathrm{k}$-th class are replaced by local means, which are used to estimate an interpolation function. After normalization, the interpolation function is assumed to be the illumination function affecting the scene. This is more precisely defined as follows. Let $G, F$ and $I$ be specific images of $g(x, y), i(x, y)$ and $f(x, y)$ respectively. Let $F=\{f[r, c]\}$ and $G=\{g[r, c]\}$ be hierarchically defined in terms of $Z=\{z[r, c]\}$ that represents the partition of the scene in different classes. Each $z[r, c]$ is a value in $\Omega=\{1, \ldots, K\}$, where $z[r, c]=k$ means that the coordinate $[r, c]$ of a pixel belongs to the $k-t h$ class. Let $\mu=\left\{\mu_{1}, \ldots, \mu_{K}\right\}$ be the class means, and $M=\left\{m[r, c]=\mu_{k} \mid z[r, c]=k \forall k \in \Omega\right\}$ be such that $m[r, c]$ is the mean of the class the pixel at location $[r, c]$ belongs to. Let $N$ be defined with the local means of $G$ such that $E[N]=M$. Let $\hat{N}_{k}$ be the interpolation function of sampled values of $N_{k}=\left\langle N, Z=k>\right.$ greater than zero ${ }^{1}$. Then $\hat{I}[r, c]=\frac{\hat{N}_{k}[r, c]}{O_{k}}$ and $O_{k}$ is the normalization factor.

Body parts are modelled with a quadratic function. The regression equation, from which the coefficients of the interpolation function are estimated, is given by:

$$
\boldsymbol{y}_{P \times 1}=\left[\mathbf{1}_{P \times 1} \boldsymbol{r} \boldsymbol{c} \boldsymbol{r} \boldsymbol{c} \boldsymbol{r} \boldsymbol{r} \boldsymbol{c c}\right]\left[\boldsymbol{\theta}_{P \times 1}\right]+\varepsilon
$$

where $y_{i}$ is the local mean ${ }^{2}$ value $n_{k}\left[r_{i}, c_{i}\right]$ for a given band and $\left[r_{i}, c_{i}\right]$ is the location of the i-th pixel sampled, which belongs to the k-th class. $r=\left[r_{1}, \ldots, r_{P}\right]^{T}$, $\boldsymbol{c}=\left[c_{1}, \ldots, c_{P}\right]^{T}$ and $\boldsymbol{r}=\left[r_{1} c_{1}, \ldots, r_{P} c_{P}\right]^{T}, \boldsymbol{r r}=\left[r_{1} r_{1}, \ldots, r_{P} r_{P}\right]^{T}$ and $\boldsymbol{c c}=\left[c_{1} c_{1}, \ldots, c_{P} c_{P}\right]^{T}$. The normalization factor $O_{k}$ is the maximal value of $\left(\hat{G}_{k}[r, c]+\gamma\right)$ and $\gamma$ is the minimal constant added to $G_{k}[r, c]$ such that the output is positive.

This procedure can be applied to each single image band. Thus, for multispectral images, the output of the present scheme is a multi-spectrally estimated illumination function.

\footnotetext{
$\overline{1<N, Z=k}>$ is the scalar product between the tensor $N$ and the thematic map that has, in a given location the value one, if the corresponding pixel belongs to the $\mathrm{k}$-th class and the value zero otherwise.

2 in a circular window of radius 11
} 


\section{Results and Discussion}

The algorithm that estimates the illumination function requires that, for each single image, a map of an expected homogeneous region must be provided. On the other hand, the problem is precisely that the classification can not be done in a uniform way and for this reason, the illumination correction is needed. As was mentioned before, it is assumed that the images of lesions with psoriasis contain three classes: curtain, normal skin and lesion. The best candidate to be interpolated is the normal skin, because it usually appears in different parts of the image. However, there are cases for which we could consider that the whole image is pure lesion. For this reason, each set of images corresponding to the same lesion was treated separately. For the images of the first patient, it was possible to provide a rough estimation of the maps corresponding to the normal skin. These maps were the output of the second stage of the classification scheme used. However, for the remaining cases, the maps of the skin, including lesions ${ }^{3}$, were used to estimate the illumination function. The thematic maps produced in the classification scheme combined with the original images used as input for the illumination correction stage are shown in Figure 2. As it can be seen, for the cases $1 A, 1 B$ and $1 C$, the region mapping normal skin was used. For the cases $2 A$ and $3 A$, a map indicating the whole image was generated. For the cases $2 B, 2 C, 3 B$ and $3 C$ the thematic map indicating skin was selected.

The image region to be modelled was convolved with a circular window with a diameter of eleven pixels, empirically found. In order to reduce the influence of pixel values belonging to other classes in the region borders during the convolution, pixel values to be excluded from the model were previously replaced with the mean value of the data to be modelled. This was also done in order to preserve the correct shadow information of the region to be modelled. An artificial grid was placed on the whole image, and grid pixels belonging to the region to be modelled were sampled.

The estimated multi-spectral illumination functions and the corresponding estimated illumination-corrected images can be seen in Figure 3. By visual assessment of the estimated illumination function for each single image of the psoriasis image set it can be noted that the extrapolation errors mostly occur in the background, which really does not contain information of interest anyhow.

Comments based on visual assessment of the illumination functions of each group of images of the same lesion follow. It is quite obvious that a quadratic model is not very representative of an object like a hand (case $(1, A)$ ). This is reflected in the illumination function, which does not include the shadows between the fingers. Continuing with the next lesion $(1, A)$, we can observe that part of the lesion $B$ of patient 1 is covered by shadow in the original images. In spite of the illumination function being almost the same for all the captures of the

\footnotetext{
${ }^{3}$ This means the output of the first stage of the classification scheme
} 


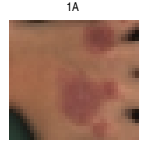

$2 \mathrm{~A}$

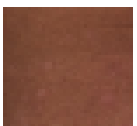

$3 A$

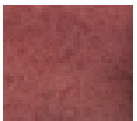

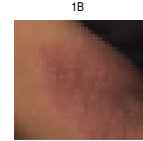

$2 B$
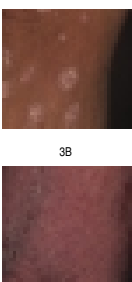

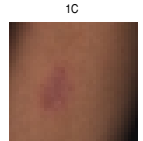

$2 \mathrm{C}$

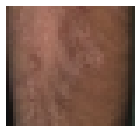

$3 \mathrm{C}$

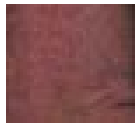

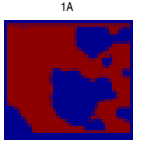

$2 \mathrm{~A}$

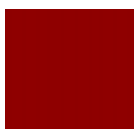

$3 A$

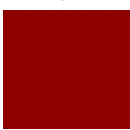

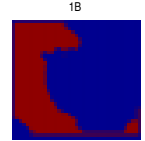

$2 B$

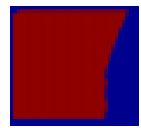

$3 \mathrm{~B}$

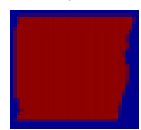

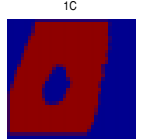

$2 \mathrm{C}$
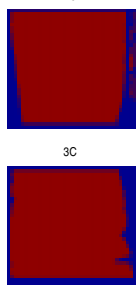

Fig. 2. Examples of original images of lesions with psoriasis (left) and the thematic maps (right) outputs of the classification scheme used in the illumination function estimation.

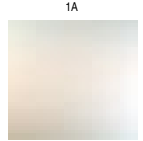

2A

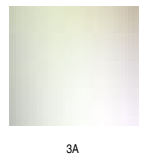

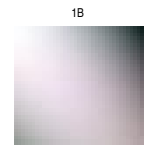

2B

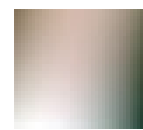

3B

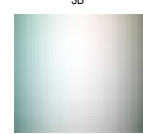

10

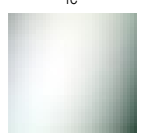

$2 C$

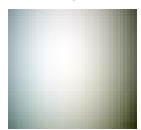

3 C

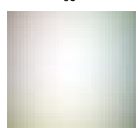

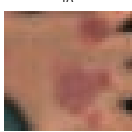

2 A

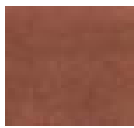

$3 \mathrm{~A}$

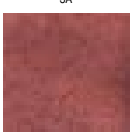

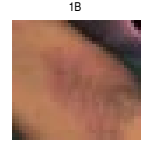

$2 B$

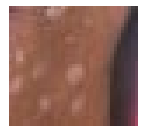

$3 \mathrm{~B}$

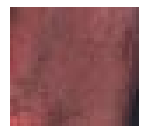

10

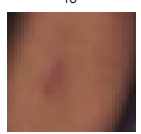

2C
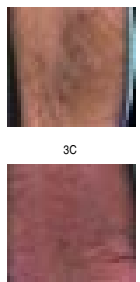

Fig. 3. Estimated illumination functions of the images of Figure 2 (left) and the corresponding illumination corrected images (right).

same case, it has to be noted, that the lesion presents occlusions in some cases, which means that, along the captures, the shadow is not affecting a given part of the lesion in the same way. With respect to lesion $C$ of patient $1-(1, C)$-, it seems that the illumination correction would not improve future alignment and registration outputs very much. Note in the illumination corrected images, that the shadows are not completely removed. Note in [5] that the provided thematic maps indicating skin do not include the regions covered by shadows. Results could be improved by finding better cost values for the discrimination function in the classification scheme. For the lesion $A$ of patient $2-(2, A)$ - it is noted that 
the illumination model represents the shadows quite well. Later on it will be shown with one example that, for this case, the illumination correction is clearly necessary. Looking at the illumination corrected images of patient 2 , lesion $B$, $-(2, B)$ - it can be noted that the illumination model does not correct the shadows in some captures well. This is due to the fact that the thematic map of the skin does not include parts of the shadows, which were assigned to the class curtain in the first classification stage: changes in the cost values could improve the results obtained. For the case $(2, C)$, it is noted that the classification output improves when the illumination is corrected. In spite of being extrapolation errors in the curtain, they obviously do not affect the classification between normal skin and lesion. The illumination functions of the case $(3, A)$ seem to represent the shadows on the original images well. This case is quite difficult, because it is hard to identify corresponding points in the images by visual assessment. Therefore, to evaluate the classification output is difficult. However, for some pairs of sessions, some degree of repeated structure can be observed. The classification output within sessions after illumination correction of the images of the case $(3, B)$ does not look very convincing. This may be due to the spherical shape of the illumination function, which is not representative of the body part modelled. In many of the illumination corrected images not shown one notes the light areas on the image corners. A better thematic map of the region to be modelled could improve the results obtained. For the case $(3, C)$, skin displacements attained after folding the elbow allow to identify corresponding points visually. By comparing the classification outputs after illumination correction within sessions, in general, they contain a repeated pattern. However, for some pairs of sessions, particularly the last ones, is not quite obvious what the correspondence should be.

The same thematic maps indicating skin generated in the previous classification using the original images, were assumed to be the output of the first classification stage using the images with illumination correction. In order to avoid extrapolation errors, the discrimination function was computed for each single image separately.

The description of an example shown in Figure 4 follows. In the first row, the $|B-G|$ bands of the original images taken for the case $2 A$ during the first session are shown. These images are clearly affected by shadows on the sides, which influences the bad corresponding lesion classification result shown in the second row. In order to be able to register the lesions, it is quite important first, that the classification output correctly maps the lesions, and second, that it contains a repeated pattern to be registered, which is not the case here. Note how the classification output improves (fifth row) after estimating the corresponding illumination functions (third row), and classifying the illumination corrected $|B-G|$ bands (fourth row). 

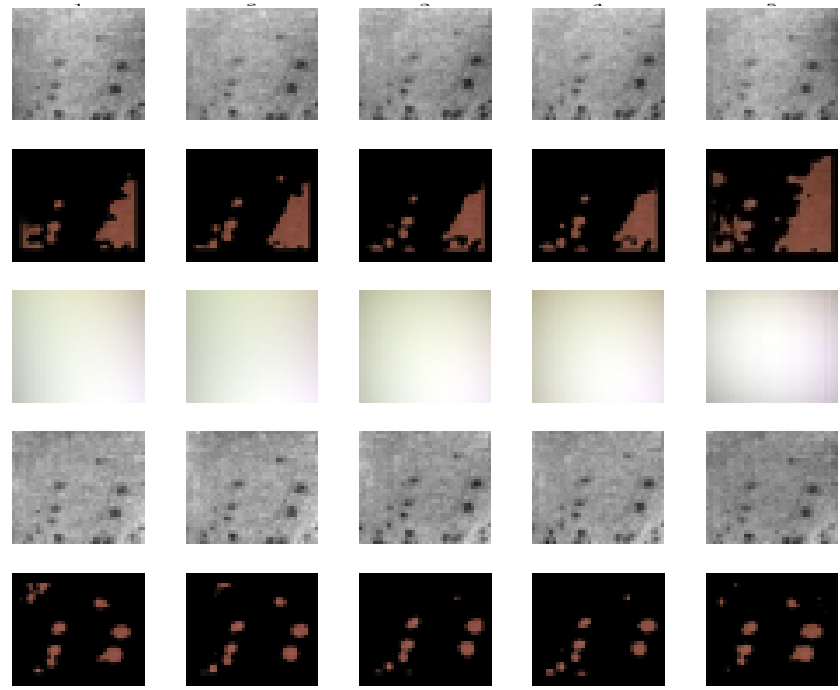

Fig. 4. Classification output before and after illumination correction.

As it can be seen, thematic maps of some lesions, have some repeated patterns in common. Based on this, it is considered that the output of the illumination correction is a better estimation of the expected data. However, in order to be able to automatically select corresponding objects in the thematic maps of the same lesion, further work needs to be done ${ }^{4}$. Since it is not the main objective of the present work, the cases for which it was not possible to automatically define corresponding objects, manual selection after visual inspection of the images is suggested.

It should to be mentioned that in many cases, shadows in the skin were assigned to the class curtain. This did not allow the introduction of the correct slope of the surface that represents the skin in the illumination model. Finding better cost values to use in the discrimination functions is encouraged in order to improve the obtained results.

\section{Conclusions}

A new scheme for illumination correction in dermatological data that combines conventional tools has been proposed. The classification scheme used has been shown to produce suitable thematic maps to be used in the estimation of the illumination function affecting the scene. It has also been shown that the illumination corrected images are a better input for the second stage of the classifica-

\footnotetext{
${ }^{4}$ See, for instance, [8], in which a shell-shaped structure of the skin chromaticities cluster was measured and modelled.
} 
tion scheme. After illumination correction, the classification results improved in cases for which it was not possible to produce thematic maps with a repeated pattern previously. By visual assessment of the results, it is tempting to extend the scheme to other application areas.

\section{Acknowledgments}

To the SITE Project funded by a grant from the Danish Technical Research Foundation (Project Number STVF 56-00-0123) for supporting the present work. To the dermatologists Lone Skov and Bo Bang of the Gentofte Hospital of Denmark and to the anonymous patients, for their collaboration during the image acquisition sessions.

\section{References}

1. Videometer. http:www.videometer.dk.

2. K. Conradsen. En Introduktion til Statistik. Bind 2. IMSOR DTH., Lyngby, 1984.

3. J. Lira. Introducción al Tratamiento Digital de Imágenes. Instituto Politécnico Nacional, Universidad Nacional Autónoma de México, Fondo de Cultura Económica., México D.F., 1 edition, September 2002.

4. G. Maletti and B. Ersbøll. A combined alignment and registration scheme of psoriasis lesions images. International Journal in Information Sciences, 2003. Accepted.

5. G. Maletti and B. Ersbøll. A hierarchical classification scheme of psoriasis images. Technical Report 6, Department of Informatics and Mathematical Modelling. Technical University of Denmark., Kgs. Lyngby. Denmark., March 2003.

6. G. Maletti, B. Ersbøll, and K. Conradsen. A contextual classifier that only requires one prototype pixel for each class. IEEE Transactions on Nuclear Science, 49(3):700-706, June 2002.

7. U. Mattsson, A Jönsson, M. Jontell, and J. Cassuto. Digital image analysis (dia) of color changes in human skin exposed to standardized thermal injury and comparison with laser doppler measurements. Computer Methods and Programs in Biomedicine, 50:31-42, 1996 .

8. M. Soriano, B. Martinkauppi, S. Huovinen, and M. Laaksonen. Skin detection in video under changing illumination conditions. $15^{\text {th }}$ International Conference on Pattern Recognition, 1:839-842, 2000.

9. Y. Wang and T. Adali. Signal Processing for Magnetic Resonance Imaging and Spectroscopy. Stocastic Model Based Image Analysis, chapter 14, pages 1-34. 2000. (Topics in Biomedical Imaging Course Notes.). 